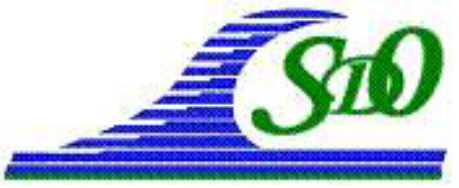

XI ìmes Journées Nationales Génie Côtier - Génie Civil

Les Sables d'Olonne, 22-25 juin 2010

DOI:10.5150/jngcgc.2010.078-D C Editions Paralia CFL

disponible en ligne - http://www.paralia.fr - available online

\title{
Utilisation d'essences alternatives de bois durables pour les ouvrages maritimes et côtiers
}

\section{Sébastien DUPRAY ${ }^{1}$, John WILLIAMS ${ }^{2}$, Jonathan SIMM $^{3}$, Melanie MEADON $^{4}$}

1. CETMEF, France (HR Wallingford à la date des travaux). sebastien.dupray@developpement-durable.gouv.fr

2. TRADA. U.K. jwilliams@trada.co.uk

3. HR Wallingford. U.K. jds@hrwallingford.co.uk

4. Environment Agency. U.K. melanie.meaden@environment-agency.wales.gov.uk

\section{Résumé :}

Les bois tropicaux sont utilisés dans les ouvrages maritimes et côtiers. Des caractéristiques élevées (durabilité, résistance mécanique, résistance à l'abrasion, résistance aux xylophages...) sont requises dans ce type d'environnement. Certains bois tempérés pourraient atteindre le niveau requis, mais uniquement s'ils étaient traités industriellement. La récente législation européenne a restreint le nombre de traitements utilisables dans le milieu maritime. De plus, il y a un souci général sur le caractère potentiellement néfaste de ces produits pour l'environnement.

Les retours sur la performance en service de certaines essences sont bons. Ainsi, les maîtres d'œuvre ou les entreprises tendent à limiter leur choix à celles-ci, notamment l'azobé et le greenheart. Par conséquent, le caractère soutenable de l'exploitation de ces seules espèces est critiquable.

La recherche conduite de 2007 à 2009 par Environment Agency, TRADA, HRWallingford et le CETMEF a permis d'identifier une liste d'essences de bois à partir de bases de données. Cette liste a ensuite été progressivement réduite à partir d'essais innovants de qualification de la résistance à l'usure et à la résistance au xylophage (en laboratoire et in situ). Enfin des caractéristiques en vraie grandeur ont été déterminées pour cinq essences.

\section{Mots clés :}

Ouvrages côtiers - Ouvrages maritimes - Bois - Xylophages - Usure - Azobé Greenheart

\footnotetext{
Abstract:

Tropical hardwoods are used in coastal and maritime structures. High level of characteristics and performance (durability, strength, resistance to abrasion, resistance to marine borers...) are expected in such environment. Some softwood of Europe could
} 
meet the requirement but only if they were treated industrially. There is some concern that preservatives may be harmful to the environment and the recent legislation however limits their use preservatives in the marine environment. Feed-back from experience of in service exposure is good for some hardwoods. Hence, designer and contractors tend to limit their choice to these only timbers, in particular greenheart and ekki. Consequently, the sustainability of these only species is questionable.

The research conducted from 2007 to 2009 by Environment Agency, TRADA, HRWallingford and CETMEF identified a list of species from available database, which was progressively reduced thanks to innovative tests to assess resistance to abrasion and to marine borers attack (laboratory tests and field tests). Finally, the full-scale strength of five species was determined.

Key words:

Coastal structures - Maritime structures - Timber - Marine borers - Abrasion - Ekki Greenheart

\section{Eléments de contexte}

\subsection{Les enjeux}

Le bois est utilisé depuis longtemps dans de nombreux ouvrages côtiers, maritimes et portuaires, notamment parce que c'est une ressource renouvelable mais aussi et surtout disponible. Le bois peut constituer la majeure partie de l'ouvrage, comme c'est le cas pour certains épis, écrans chasse-mer, certaines estacades ou certaines portes par exemple. Mais, il peut aussi constituer des parties spécifiques de certains ouvrages comme des pieux de fondation, des défenses d'accostage, des lisses d'approches, des parties de portes ou des platelages par exemple.

Dans tous les cas, l'utilisation de bois a toujours été difficile notamment à cause de l'agressivité particulière de l'environnement maritime et côtier exacerbée pour certains types d'ouvrages (en particulier les ouvrages de défense contre la mer), à cause de difficultés liées à certaines techniques de construction (comme la battage) et à cause de la position dans la zone de marnage.

Par conséquent et pour construire des ouvrages durables, des essences de bois avec des caractéristiques élevées sont recherchées, notamment avec de grandes longueurs et de grandes sections disponibles, une stabilité géométrique aux cycles humidité / séchage, une résistance mécanique élevée, une résistance élevée aux attaques biologiques (xylophages et champignons), une résistance élevée à l'usure induite par les vagues et les sédiments mis en suspension et dans certains cas une résistance permettant la mise en œuvre par battage. Un équilibre ad hoc doit être recherché pour ces différentes propriétés essentielles du bois (CROSSMAN \& SIMM, 2004).

Les mécanismes qui peuvent conduire à une réduction de la section des éléments structuraux sont importants, en particulier s'ils concernent des éléments critiques 
comme les pieux (NGUYEN et al., 2008a ; NGUYEN et al., 2008b) ou les poutres maîtresses. En effet, ils peuvent in fine induire des dommages voire la ruine de l'ouvrage qui ne pourrait plus supporter les charges auxquels il est exposé. A ce titre, la résistance à l'abrasion et la résistance aux xylophages sont des propriétés essentielles.

L'environnement côtier présente différents degrés d'agressivité associée aux effets des frottement et/ou des impacts de sédiments fins et grossiers (voire de galets) sur les ouvrages ou parties d'ouvrages exposés aux vagues. C'est d'autant plus vrai que l'ouvrage a un rôle de protection contre la mer comme les épis par exemple. Il n'existe pas pour le moment de méthode fiable pour quantifier cette agressivité. Cependant, certains s'interrogent sur l'effet que le changement climatique pourrait avoir sur les conditions d'exposition et, par conséquent, sur la performance des matériaux et la durée de vie de ces ouvrages (DUPRAY \& SIMM, 2007 ; NICHOLLS \& DE LA VEGALEINERT, 2008).

L'attaque des xylophages n'est pas aisément détectée et elle représente donc un enjeu particulier. Par ailleurs, certains craignent une augmentation du risque d'attaque par les xylophages du bois. Elle pourrait être induite non seulement par l'amélioration de la qualité des eaux côtières (favorisant leur développement et leur vigueur) mais aussi par une modification des caractéristiques des eaux à la côte (notamment la température) favorisant la migration de certaines espèces de xylophages (GOLLASH, 2006). De manière schématique, les xylophages du bois sont soit du type "crustacé" (comme le Limnoria spp) soit du type "ver à bois" (comme le Teredo spp), (HALL \& SAUNDERS, 1967).

Pour ce qui concerne les parties enterrées des ouvrages en bois, en particulier les pieux, celles-ci peuvent être exposées à des agressions induites par certains sols naturels (comme les tourbes) ou certaines pollutions du sol. Les conditions anoxiques tendent à limiter les autres attaques fongiques à des dégradations de surface (RAPP et al., 2007).

La pratique a montré que peu de bois blancs (sauf s'ils sont traités) offrent des caractéristiques suffisantes pour résister aux agressions marines et côtières, en particulier pour les ouvrages sensibles ou à fort enjeu. Ainsi, quelques essences de bois exotiques ont été historiquement largement utilisées car elles offrent des caractéristiques techniques acceptables, une faible maintenance mais ces bois d'origine parfois très lointaine sont importés (BRAZIER, 1995). Par ailleurs, l'expérience a montré que la bonne performance d'une essence dans une application terrestre n'implique pas nécessairement une performance suffisante pour des applications côtières ou maritimes. Ainsi, on a recours à l'azobé et le greenheart qui présentent des caractéristiques dimensionnelles, de résistance et de durabilité ad hoc même si on peut parfois aussi noter l'utilisation d'autres essences comme le balau, le jarrah, ... . 

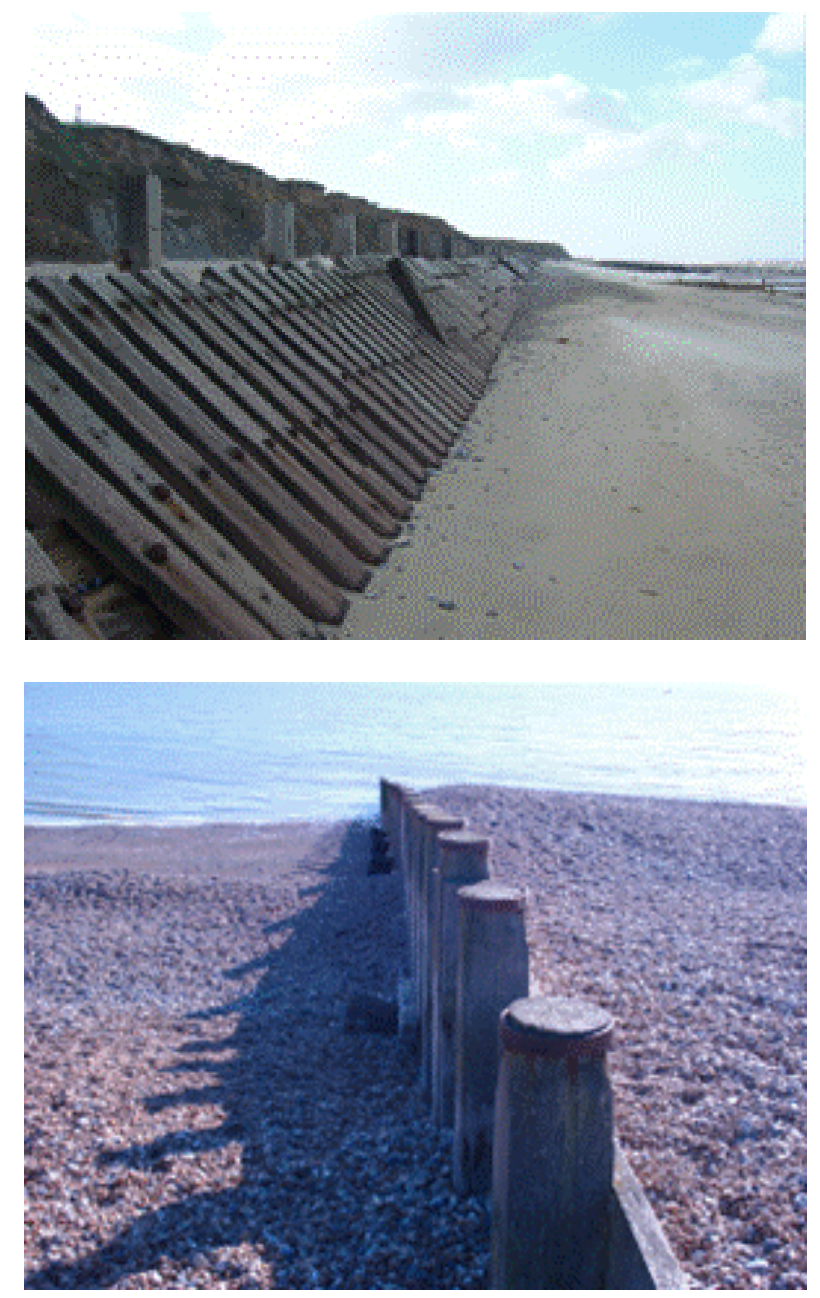

Figure 1. Exemple d'utilisation de bois durable en milieu côtier dans des ouvrages de protection du littoral (source CETMEF) ou dans des épis imperméables (source HRWallingford).

\subsection{Les bois tropicaux sont-ils la bonne réponse?}

Il est pourtant légitime de s'interroger sur la pertinence d'utiliser les bois tropicaux mentionnés plus haut, qui sont exploités à l'étranger et transportés sur de longues distances.

Tout d'abord, l'utilisation de bois blancs pour ces applications requiert des traitements dans la masse (par exemple à base de cuivre, de chrome ou d'arsenic). Les traitements de surface (comme à base de créosote) n'offrent pas de garantie de pérennités suffisante dans les ouvrages exposés à l'abrasion, qui pourrait éroder cette couche protectrice. Les récentes réglementations communautaires (WILLIAMS et al., 2004a) ont conduit à de fortes limitations sur les traitements du bois ainsi que l'obligation de gérer la fin de vie des bois traités. Ainsi, l'industrie se retourne plus fortement vers des bois non traités et donc vers des essences tropicales. 
Ensuite, les critères de sélection des essences de bois pour les ouvrages maritimes restent très empiriques malgré l'apparition de certains codes ou certaines recommandations notamment à l'étranger. Ainsi, on peut constater un certain conservatisme qui conduit à restreindre les choix entre quelques essences par défaut ayant fait preuve de bonnes performances en service, comme l'azobé et le greenheart. Cela conduit de fait à la sur-utilisation de ces meilleures essences, et vraisemblablement à leur surexploitation ainsi qu'à une surévaluation des prix sur le marché. L'utilisation d'autres essences permettrait d'une part de diminuer la pression sur les rares essences utilisées et d'autre part de limiter l'utilisation des essences par défaut aux situations où il n'y a pas d'alternative.

Enfin, on connaît bien certains risques associés à l'achat de bois tropicaux qui sont particulièrement la légalité de leur origine, la qualité des conditions d'exploitation de la forêt sur place ainsi que la traçabilité. Le maître d'ouvrage ou le maitre d'œuvre devrait demander des preuves documentées de la légalité du bois, des bonnes conditions d'abattage ainsi que des éléments relatifs à sa traçabilité au fournisseur. Par ailleurs, l'existence de certaines marques et le recours à celles-ci permet de minimiser ces risques; on peut notamment citer les marques suivantes en notant bien qu'elles n'ont pas toutes strictement le même champ d'application : OLB (Origine Légale du Bois), FSC (Forest Stewardship Council for legality), PEFC (Programme for the Endorsement of Forest Certification Schemes for legality and sustainability), SFI (Sustainable Forest Initiative for legality and sustainability), CSA (Canadian Standard Association for legality and sustainability).

\subsection{Vers l'identification d'essences alternatives à l'azobé et au greenheart}

La figure 2 présente un logigramme général avec une succession de questions permettant trier (ou de choisir) des bois tropicaux pour les applications maritimes et côtières. Il est adapté de démarches présentées dans la littérature (CROSSMAN \& SIMM, 2004 ; SIMM et al., 2004) et a pour objectif de mettre en perspective le travail présenté dans cet article qui porte sur les essences moins utilisées. Les critères portent ici sur le recyclage, la légalité, la soutenabilité de l'exploitation, la disponibilité et les caractéristiques techniques. Ces dernières sont la longueur, la section, la résistance mécanique et la durabilité ; elles sont déterminées (ou déterminables) par des essais, des pilotes ou des retours d'expérience. Elles doivent être adaptées au projet d'ouvrage ainsi qu'à l'environnement sur son site.

En application de ce processus de tri, on peut trier le bois en différentes catégories, notamment les catégories "inapproprié" et "généralement utilisé". Dans certains cas, il ne sera pas possible de conclure par manque de données techniques ou de retour d'expérience. 


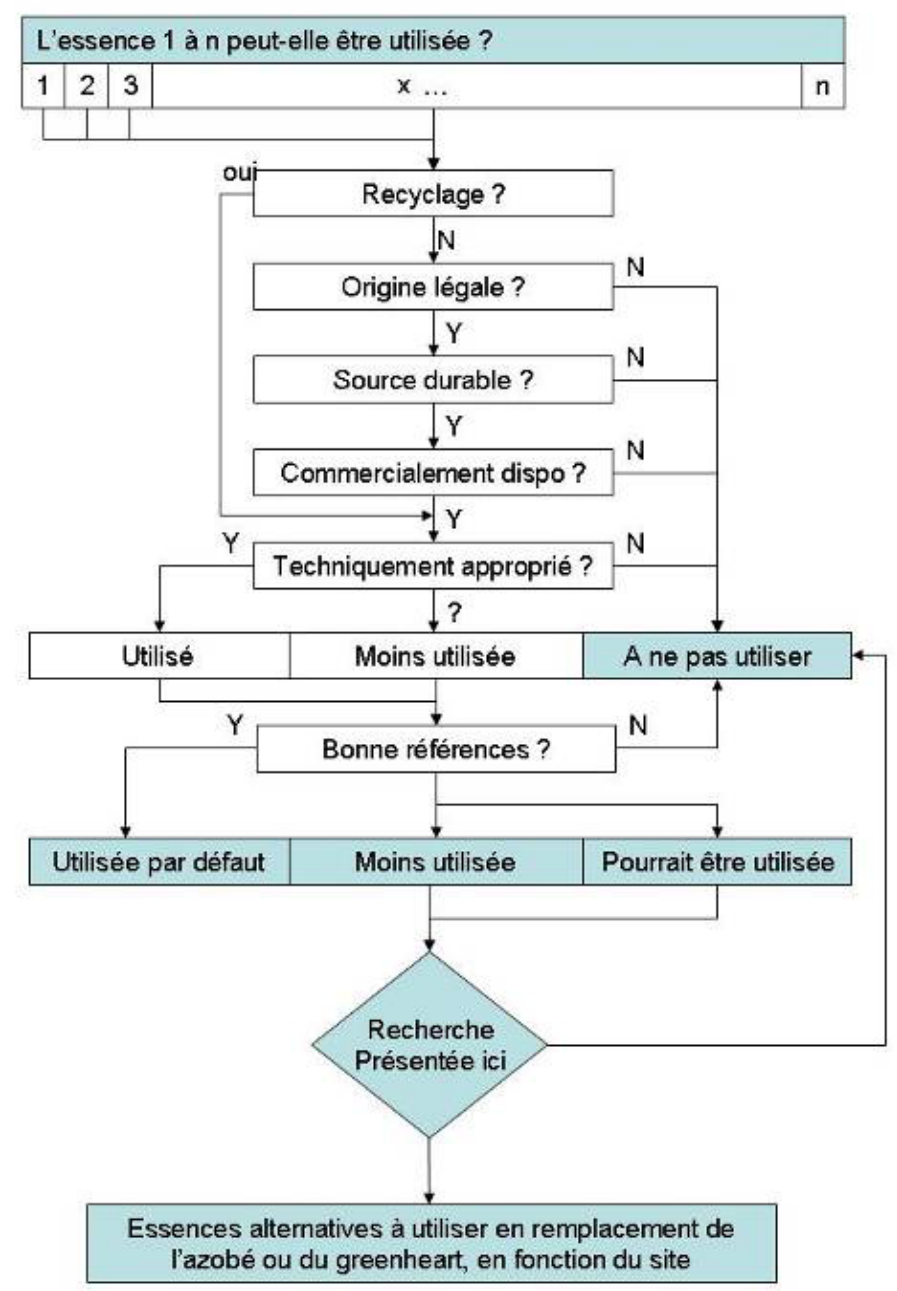

Figure 2. Principe de sélection des essences et positionnement de la recherche dans cette démarche.

\section{Approche adoptée pour identifier puis sélectionner les essences alternatives durables}

Cette section présente brièvement la méthode qui a permis d'identifier des essences moins utilisées et/ou moins connues, ainsi que le processus d'estimation de leur adaptation à l'environnement des ouvrages maritimes et côtiers. Quatre étapes ont été suivies :

a) Étape 1: identification des essences prometteuses à partir des informations disponible ou de l'expérience de terrain; sélection d'essences connues pour servir de référence pour les essais ultérieurs ;

b) Étape 2: réduction de la liste grâce à des essais accélérés d'exposition aux xylophages et à des essais d'usure au laboratoire (voir Section 2.2.1 et 2.2.2 cidessous) ; 
c) Étape 3 : essais mécaniques sur les essences choisies par référence à la norme EN408; les essais sont conduits sur une liste limitée (les essences non testées dans ce projet pourront l'être dans un autre projet ou dans une suite de ce projet) ;

d) Étape 4 : pilote et exposition d'essences à des conditions réelles d'exposition.

\subsection{Identification d'une liste d'essences alternatives}

Une liste d'essences a été compilée à partir des données disponibles dans la littérature et des connaissances propres aux partenaires du projet. Il s'agit des travaux réalisés pour la rédaction du "Manual on the use of timber in coastal and fluvial engineering" (CROSSMAN \& SIMM, 2004) : 41 essences, du rapport de Biodiversity International Ltd (BIODIVERSITY INTERNATIONAL, 2007): 19 essences, des essences investigués par les fournisseurs membres du projet (AITKEN \& HOWARD, 2007 ; ECOCHOICE, 2007), de l'expérience de TRADA published (OLIVER, 1974; METTEM \& RICHENS, 1991), des bases de données PROSPECT et de l'Oxford Forestry Institute reprises par le BRE (MAUN, 2000), de la base de données de l'US Department of Agriculture Forest Products Laboratory (CHUDNOFF, 1979) et de la base de données Tropix 5 du CIRAD (BOLZA \& KEATING, 1972; MAINIERI \& CHIMELO, 1989).

Cette liste de plus de 50 essences a été réduite pour permettre de focaliser l'effort du projet sur les essences les plus prometteuses et le résultat est présenté au tableau 1. Pour ce faire, les essences répondant aux critères d'exclusion ci-dessous n'ont pas fait l'objet d'investigations complémentaires :

a) Qualité ou valeur commerciale rendant l'essence plus vraisemblablement utilisées dans d'autres applications à plus forte valeur ajoutée (mobilier, huisserie, construction, ...);

b) Connaissance de très mauvaise résistance à l'attaque par les xylophages ou à l'usure ;

c) Connaissance d'une très faible résistance mécanique ou d'une mauvaise performance pour d'autres modes de sollicitation (eg. déformation par humidité/séchage).

Les essences dont certaines propriétés sont connues et publiées ont été conservées et identifiées comme "élément de comparaison générale" ; l'azobé et le greenheart ont été identifiés comme "essences de référence" car elles sont bien connues pour leur bonne adaptation au milieu maritime et côtier.

\subsection{Présélection des essences}

Pour utiliser de manière fiable les résultats de cette recherche, il était essentiel de concentrer l'effort sur des essences qui pourraient in fine être utilisées avec confiance. Ainsi, des essais 'cribles' ont été réalisés afin d'éliminer à partir d'essais accélérés les essences qui présentent des performances faibles pour les propriétés critiques. Pour ce faire, des échantillons ont été préparés à partir des stocks disponibles et exposés à deux essais de laboratoire et un essai in situ. 
Thème 5 - Ouvrages portuaires, côtiers et offshore

Tableau 1. Synthèse des essais de sélection des essences alternatives.

\begin{tabular}{|c|c|c|c|c|c|c|c|c|c|}
\hline \multirow[t]{2}{*}{ Nom commercial } & \multirow[t]{2}{*}{ Nom botanique } & \multirow[t]{2}{*}{$B / R$} & \multirow[t]{2}{*}{$\boldsymbol{E A}$} & \multicolumn{2}{|c|}{$\begin{array}{l}\text { Résistance } \\
\text { au Limnoria }\end{array}$} & \multicolumn{2}{|c|}{$\begin{array}{l}\text { Resistance } \\
\text { à l'usure }\end{array}$} & \multicolumn{2}{|c|}{$\begin{array}{l}\text { Résistance } \\
\text { au taret }\end{array}$} \\
\hline & & & & $G$ & $E$ & $G$ & $E$ & $G$ & $E$ \\
\hline Azobé ** & Lophira alata & $x$ & & $(-)$ & & ++ & & $\approx$ & \\
\hline Greenheart ** & Chlorocardium rodiei & $x$ & & & $(+)$ & & - & & $\approx$ \\
\hline Balau & Shorea spp. & $x$ & & ++ & ++ & -- & - & ++ & ++ \\
\hline Opepe (bilinga) & Nauclea diderrichii & $x$ & & $(-)$ & $(-)$ & -- & -- & $\approx$ & $\approx$ \\
\hline Pin Douglas & Pseudotsuga menziesii & $x$ & & $(+)$ & $(+)$ & $(-)$ & - & - & - \\
\hline Pin sylvestre *** & Pinus sylvestris & $x$ & & -- & -- & $\cdot$ & $\cdot$ & - & -- \\
\hline Karri & Murraya koenigii & $x$ & & $(-)$ & $(-)$ & $(-)$ & - & - & - \\
\hline Purpleheart & Peltogyne spp & $x$ & & -- & -- & $(-)$ & - & -- & -- \\
\hline Alep & Desbordesia glaucescens & & $x$ & - & $(-)$ & ++ & $(+)$ & - & -- \\
\hline Angelim vermelho & Dinizia excelsa & & $x$ & -- & $(-)$ & $(-)$ & -- & ++ & ++ \\
\hline Basrolocus & Dicorynia guianensis & & $x$ & $(-)$ & $(-)$ & $(-)$ & -- & $\approx$ & $\approx$ \\
\hline Cloeziana & Eucalyptus cloeziana & & $x$ & $(-)$ & $(-)$ & $(-)$ & -- & ++ & ++ \\
\hline Cupiuba & Goupia glabra & & $x$ & ++ & ++ & -- & - & ++ & ++ \\
\hline Dabéma (dahoma) & Piptadeniastrum africanum & & $x$ & $(-)$ & $(+)$ & $(-)$ & -- & $\approx$ & $\approx$ \\
\hline Evuess & Klainedoxa gabonensis & & $x$ & -- & -- & ++ & $(+)$ & ++ & ++ \\
\hline Garapa & Apuleia leiocarpa & & $x$ & $(+)$ & $(+)$ & $(-)$ & -- & - & - \\
\hline Massaranduba & Manilkara spp & & $x$ & -- & $(-)$ & -- & -- & $\approx$ & $\approx$ \\
\hline Mora & Mora excelsa & & $x$ & $(+)$ & $(+)$ & $(-)$ & -- & $\approx$ & $\approx$ \\
\hline Mukulungu & Austranella congolensis & & $x$ & -- & -- & $(+)$ & $(-)$ & ++ & ++ \\
\hline Niové & Staudtia kamerunensis & & $x$ & ++ & ++ & $(-)$ & -- & ++ & ++ \\
\hline Okan & Cylicodiscus gabunensis & & $x$ & $(-)$ & $(-)$ & $(-)$ & -- & ++ & ++ \\
\hline Piquia & Caryocar glabrum & & $x$ & $(+)$ & ++ & $(-)$ & -- & $\approx$ & $\approx$ \\
\hline Sapucaia & Lecythis paraensis & & $x$ & $(-)$ & $(+)$ & $(+)$ & $(-)$ & $\approx$ & $\approx$ \\
\hline Sougé & Parinari excelsa & & $x$ & $(-)$ & $(-)$ & ++ & $(+)$ & $\approx$ & $\approx$ \\
\hline Tali (missanda) & Erythrophleum ivorense & & $x$ & -- & $(-)$ & ++ & $(+)$ & $\approx$ & $\approx$ \\
\hline Tatajuba & Bagassa guianensis & & $x$ & $(+)$ & $(+)$ & $(+)$ & $(-)$ & ++ & ++ \\
\hline Timborana & Enterolobium schomburgkii & & $x$ & $(-)$ & $(+)$ & $(+)$ & $(-)$ & ++ & ++ \\
\hline \multicolumn{10}{|c|}{$\begin{array}{l}\text { Note: acaria quara (Minquartia guianensis), araracanga (Aspidosperma megalocarpum), afina (Strombosia } \\
\text { glaucescens), aweimfo samina (Albizia ferruginea), ayan (Distemonanthus benthamianus), bompagya (Mammea } \\
\text { Africana), favinha prunhela (Enterolobium schomburgkii), mata-Mata (Eschweileria odora), muiracatiara } \\
\text { (Astronium le-cointei), Uchi torado (Sacoglottis guianensis), wonton (Morus mesozygia) n'ont pas été testées dans } \\
\text { le cadre de ce programme car elles n'étaient pas commercialement disponibles. Cependant elles semblent pouvoir } \\
\text { être des essences alternatives. }\end{array}$} \\
\hline
\end{tabular}

\subsubsection{Essai 1: attaque par des xylophages au laboratoire}

La norme EN 275 sur l'attaque du bois par les xylophages est un moyen normalisé d'apprécier la résistance aux attaques des xylophages mais elle présente un intérêt majeur qui est sa durée de réalisation. Ainsi, une méthode accélérée d'identification des essences qui ne passeraient pas l'essai EN275 a été mise au point par BORGES et al. (2003) Cette méthode innovante consiste à exposer des prises d'essai à des xylophages (Limnoria quadripunctata Holthuis). La résistance de l'essence testée est déterminée à 


\section{XI $I^{\text {èes }}$ Journées Nationales Génie Côtier - Génie Civil Les Sables d'Olonne, 22-25 juin 2010}

partir du rythme d'alimentation via la comptage des pellettes de défécation du crustacée. Ce rythme d'alimentation est ensuite comparé à celui observé pour l'azobé et le greenheart.

Les crustacées (Limnoria quadripunctata Holthuis) sont obtenus par culture sur du pin sylvestre, qui est aussi utilisé pour s'assurer de leur vigueur avant de les mettre en contact avec la prise d'essai. Un limnoria est transféré dans chaque cellule qui contient une prise d'essai $(20 \mathrm{~mm} \times 4.5 \mathrm{~mm} \times 2 \mathrm{~mm})$ et qui est maintenu dans des conditions de vie du crustacée (voir figure 3). 5 prises d'essais sont testées par essence. Les essais ont été réalisés entre l'hiver 2007 et le printemps 2008. Les résultats sont présentés de manière synthétique au tableau 1.

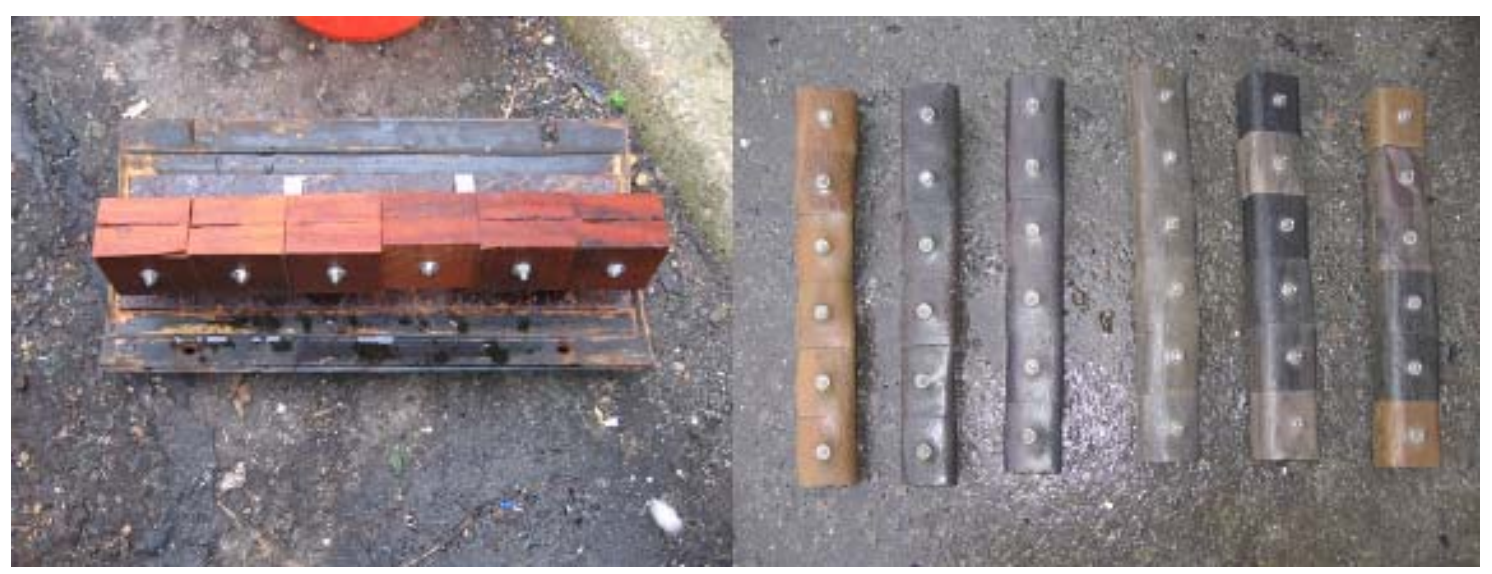

Figure 3. Vue des prises d'essai avant et après l'exposition à 16000 tours dans le tambour contenant l'eau et les sables/graviers (source TRADA).

\subsubsection{Essai 2: usure au laboratoire}

Il n'existe pour le moment aucun essai permettant de mesurer dans des conditions normalisées la résistance du bois à l'usure pour des applications dans des ouvrages maritimes ou côtiers. Un essai accéléré a été développé par SAWYER et WILLIAMS (2005) qui consiste à exposer dans un tambour rotatif des prises d'essais à l'action d'un mélange d'eau, de sable et de gravier. Chaque essence est représentée par 2 prises d'essai de $75 \mathrm{~mm} \times 80 \mathrm{~mm} \times 50 \mathrm{~mm}$ qui ont précédemment exposées à un lent courant d'eau de mer pendant 4 semaines.

La résistance à l'usure est estimée à partir de la perte de masse (voir figure 4) et les résultats sont comparées avec la perte de masse de l'azobé et du greenheart. Les résultats sont présentés de manière synthétique au tableau 1.

\subsubsection{Essai 3: exposition in situ aux xylophages du bois}

Les essences ayant passé avec succès les essais 1 et 2 sont soumises au protocole de la norme EN275. Une modification est apportée au protocole (qui demande une immersion 
verticale) en immergeant les prises d'essais à l'horizontale afin qu'ils soient tous exposés aux mêmes conditions d'immersion et de marnage, en particulier à marée basse. Le site d'immersion est le lagon Ria Formosa (Portugal). Le site est situé sur l'Atlantique et offre de bonnes conditions d'exposition aux xylophages (WILLIAMS et al., 2004a) : température de l'eau entre $12^{\circ} \mathrm{C}$ et $28^{\circ} \mathrm{C}$; une salinité entre 33 et $36.5 \mathrm{~g} / \mathrm{kg}$ entre l'hiver et l'été (NEWTON \& MUDGE, 2003); marée entre 1.35 m (morte eau) à $3 \mathrm{~m}$ (vive eau).

Pour chaque essence (y compris azobé et greenheart, ainsi que les essences pour comparaison), six prises d'essais $(20 \mathrm{~mm} \times 75 \mathrm{~mm} \times 200 \mathrm{~mm})$ sont préparées et immergées sur le site (voir figure 5). Une "planchette" de contrôle en pin sylvestre est aussi immergée pour s'assurer de l'activité des xylophages sur le site.

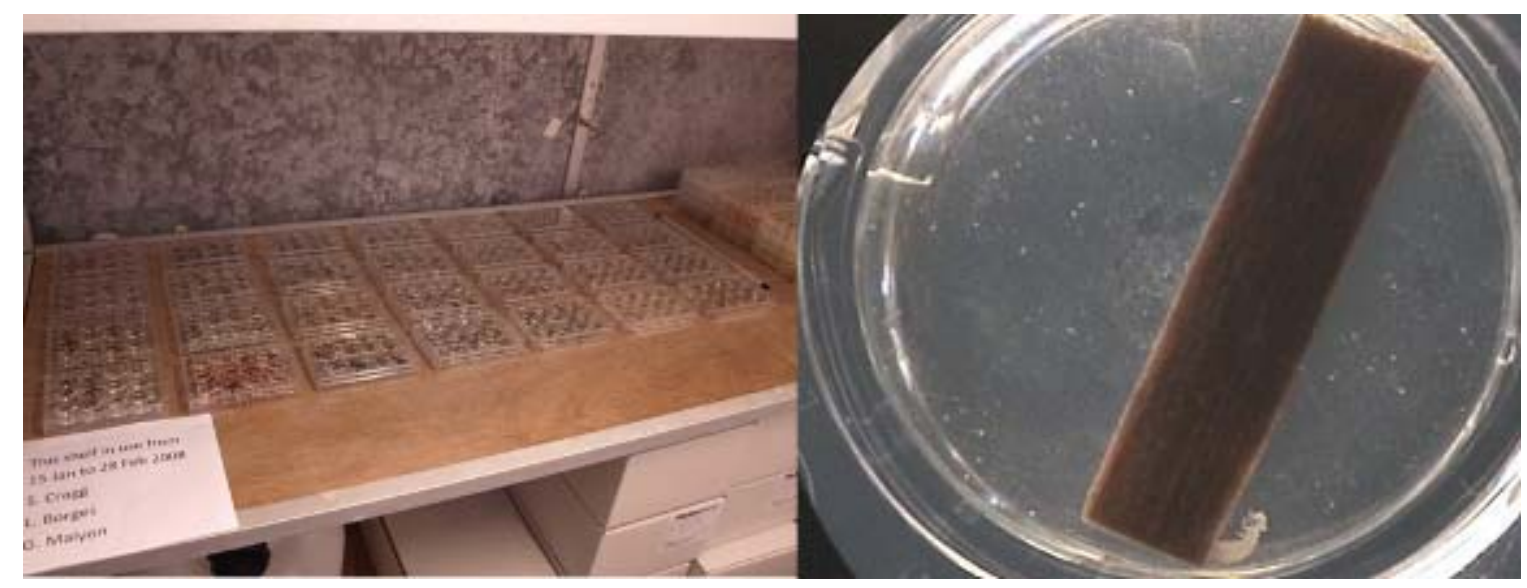

Figure 4. Vue des cellules utilisées pour mettre en présence un Limnoria et une prise d'essai ; zoom sur une prise d'essai de greenheart intacte (source TRADA).

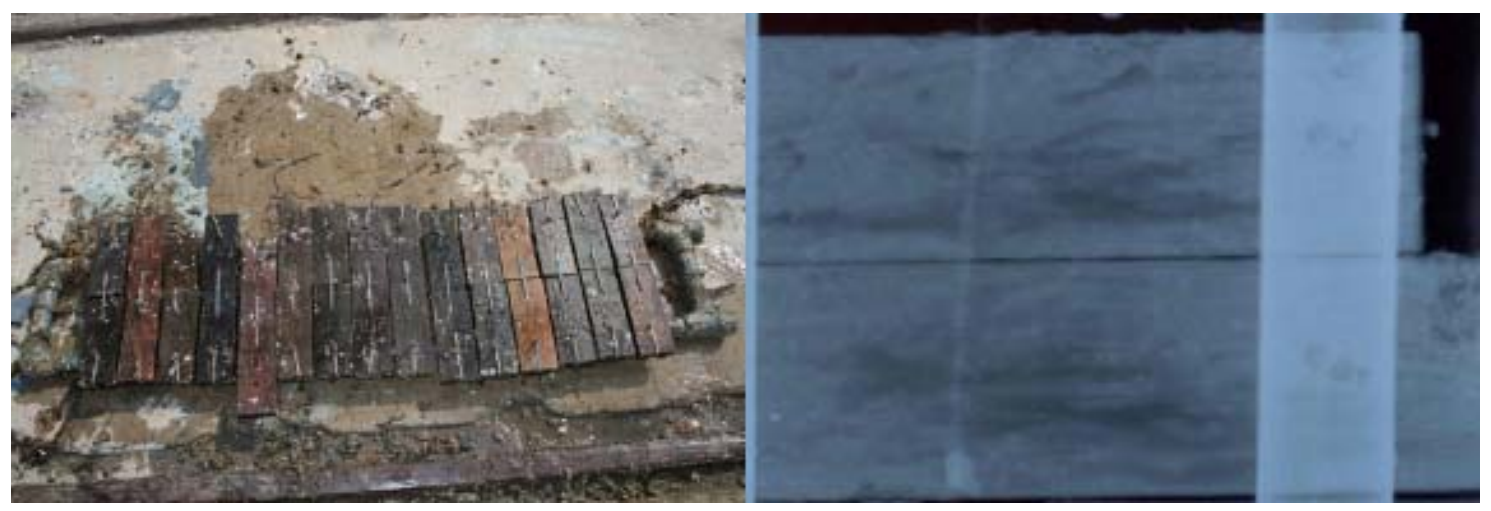

Figure 5. Vue des planchettes utilisées comme prise d'essai pour l'exposition aux xylophages in situ sur le site de Ria Formosa; vue des tunnels creuses par les tarets à l'aide de rayons $X$ (source TRADA).

Après une saison d'immersion, les planchettes sont examinées pour rechercher des signes visuels d'attaques de crustacées (WILLIAMS, 1999 ; WILLIAMS et al., 2004b). 


\section{XI $I^{\text {èes }}$ Journées Nationales Génie Côtier - Génie Civil Les Sables d'Olonne, 22-25 juin 2010}

Pour ce qui concerne l'analyse des attaques par les tarets, les planchettes sont soigneusement emballées dans des films plastiques pour éviter tout dessèchement puis passées aux rayons X pour évaluer l'étendue de l'attaque interne (voir figure 5). Les planchettes sont remises en place pour poursuivre leur immersion.

Les immersions ont été réalisées au printemps 2008 et une première analyse a été réalisée à l'automne 2008 après une saison d'exposition. L'analyse des attaques sur le pin sylvestre a montré que l'attaque par les crustacées a été effective mais modérée pendant cette saison. Les résultats sont présentés de manière synthétique au tableau 1.
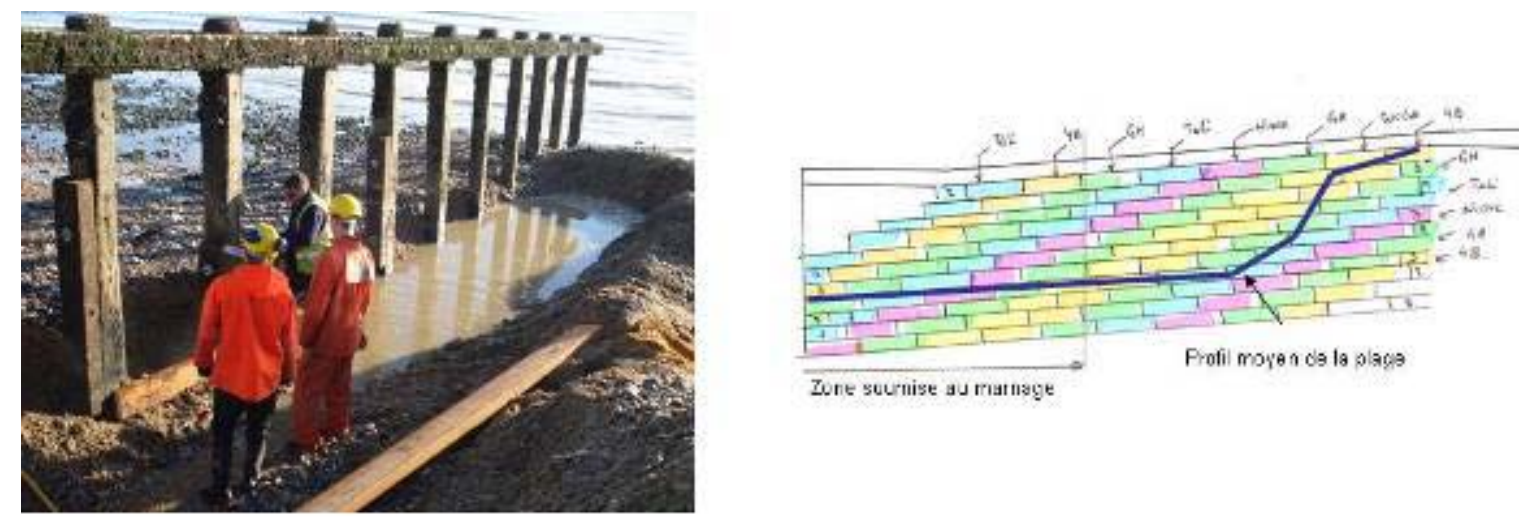

Figure 6. Vue de l'épi pilote avant sa reconstruction avec des essences alternatives (gauche) et du plan d'implantation des essences (droite).

\subsection{Analyse des résultats de la présélection.}

Le tableau 1 présente de manière synthétique les résultats de la présélection, en indiquant les essences par leur nom commercial et botanique. En effet, la qualité du bois peut varier avec le terroir (conditions de sols, de climat, ...) le nom commercial du bois permet d'identifier un terroir particulier pour un même nom botanique.

Les essences qui n'ont pu être testées car elles n'étaient pas commercialement disponibles ou car le temps d'approvisionnement à partir d'exploitations forestières était trop long (même si elles pourraient offrir une alternative potentielle) sont aussi indiquées en bas de tableau. Dans le tableau 1, une distinction a été faite pour les essences qui présentent une différence statistiquement significative (déterminée par une analyse de la variance des populations de résultat) par rapport à l'azobé et au greenheart par "++" ou "--". Quand une différence a été observée mais qu'elle n'est pas statistiquement significative elle a été indiquée par "(+)" or "(-)". Enfin, lorsque le nombre de prises d'essais n'était pas suffisant pour mener une analyse statistiquement correcte, les essences ne présentant pas de performance différente ont été considérées comme équivalentes et indiquées par " $\approx$ ".

La masse volumique est parfois utilisée comme un indicateur de la qualité du bois, notamment pour ce qui est de sa résistance mécanique ou à l'usure à l'état sec (GREEN, 
\& PITMAN, 2001). Cependant, les résultats de cette étude semblent montrer que cette corrélation est moins fiable pour ce qui concerne les résultats de la résistance à l'usure en milieu humide. Cela peut s'expliquer par le rôle important que joue la texture du bois en particulier à l'état humide et qui n'est pas apprécié avec la masse volumique seule.

L'analyse des résultats significatifs et par référence à l'azobé et au greenheart suggère les conclusions suivantes :

a) dans les milieux où le Limnoria est prévalent, 3 essences présentent une meilleure performance (cupiuba, niove et yellow balau) ou une performance moindre (evuess, mukulungu et purpleheart);

b) cinq essences présentent une résistance à l'usure inférieure à l'azobé et au greenheart (yellow balau, opepe, cupiuba and massaranduba);

c) aucune essence ne présente une résistance à l'usure meilleure que l'azobé et le greenheart même si 4 essences sont meilleures que le greenheart (alep, evuess, souge et tali);

d) neuf essences présentent une résistance in situ (surtout au taret du bois) meilleure (angelim vermelho, cloeziana, cupiuba, evuess, mukulungu, niové, okan, tatajuba, timborana) alors que 4 essences présentent une résistance in situ moindre (pin sylvestre, purpleheart, alep et garapa).

D'une manière générale et pour ce qui concerne la durabilité :

a) seul un petit nombre d'essences sont comparables ou meilleures que l'azobé et le greenheart. Ceci explique pourquoi la pratique a historiquement eu recours surtout à ces essences. Cependant, 5 essences (niove, sapucaia, souge, tatajuba, timborana) se détachent et méritent une attention plus poussée ;

b) puisque l'azobé présente une résistance à l'usure extrêmement élevée, l'utilisation de cette espèce comme référence tend à exclure des essences qui seraient viables pour des milieux où le risque d'usure à moyen à modéré; alors 7 autres essences pourraient être considérées (bascarolus, cloezina, dabema, garapa, mora, okan et piquia);

c) enfin, il est important de noter que les sites ne sont pas tous exposés avec le même degré de sévérité à l'ensemble des attaques des différents xylophages et à l'usure ; ainsi il est proposé que le tableau 1 puisse être un outil d'aide au choix d'essences adaptées à des sites où les risques sont identifiés. Par exemple, l'alep pourrait être utilisé dans des sites où la menace des xylophages est nulle même si le risque d'usure est bien présent.

Enfin, une analyse globale des essences, s'appuyant sur les résultats des essais de présélection / de durabilité a été menée pour choisir un nombre limité (5) d'essences sur lesquelles poursuivre les essais. Ce nombre est limité par les finances du projet. L'analyse globale et multicritère s'est appuyée sur des aspects complémentaires : la durabilité de l'approvisionnement, les régions d'origine (qui peuvent affecter les saisons de livraison), la disponibilité commerciale, la longueur disponible, les sections 
disponibles, la forme des troncs, la facilité à travailler l'essence (capacité à les travailler avec des outils de chantier), la stabilité à l'humidité/séchage.

Cette analyse multicritère a été faite collégialement et à conduit à identifier les 5 essences suivante : angelim vermelho (dinizia excelsa), cupiuba (goupia glabra), evuess (klainedoxa gabonensis), okan (Cylicodiscus gabunensis), tali ou missanda (erythrophleum ivorense).

Ces essences ont ainsi été testées en vraie grandeur pour en déterminer la classe d'usage, travaillées en atelier pour en estimer la facilité d'emploi et enfin mises en œuvre sur un site pilote. L'exposition in situ aux xylophages du bois est poursuivie pour confirmer sur une période plus longue les résultats obtenus (et présentés au tableau 1) notamment pour ce qui concerne la résistance aux Limnoria.

\section{Travaux complémentaires pour déterminer la résistance en vraie grandeur des 5 essences alternatives}

\subsection{Essais mécaniques en vraie grandeur}

La réalisation d'essais mécaniques en vraie grandeur a été précédée d'une évaluation visuelle des stocks (faite sur place en Hollande) par rapport à la présence de noeuds, de défauts du bois ou de signes de ruptures. La classification visuelle proposée par BS 5726 de 2007 a été retenue et a permis d'évaluer qu'au sein de la partie évaluée du stock évaluée, environ 10 grumes sur 160 analysées visuellement présentaient des défauts susceptibles de les déclasser.

Après réception et préparation des échantillons, des essais de flexion (quasi-statiques 4 points) ont été réalisés suivant la norme EN 408 sur 20 prises d'essais par essence. La masse volumique du bois a été déterminée par référence à $\mathrm{EN} 13183$ et sa teneur en eau par rapport à EN 384. Les prises d'essais $(0.15 \mathrm{~m} \times 0.5 \mathrm{~m} \times 3 \mathrm{~m}$ à $1 \%$ près $)$ ont progressivement été amenées à $20 \pm 2^{\circ} \mathrm{C}$ et $65 \pm 5 \%$ d'humidité dans le hall d'essai de TRADA avant l'essai. In fine, les essences ont été classées dans les catégories de la norme EN338. Les résultats sont synthétisés au tableau 2.

\subsection{Essais de mise en œuvre des essences alternatives}

L'utilisation des essences est aussi fortement liée à la capacité des opérateurs à travailler avec ces essences. Ainsi, des essais de mise en œuvre ont été réalisés dans les ateliers de British Waterways et à l'occasion de l'incorporation dans le pilote. Il n'existe pas à l'heure actuelle de norme permettant de juger de manière parfaitement objective de la qualité des essences face aux différentes opérations de mise en œuvre. Cependant, un jugement qualitatif a été porté par des opérateurs des ateliers et sur le chantier qui ont l'habitude de travailler avec du bois. La synthèse de ce jugement qualitatif est présentée dans le tableau 3. 
Thème 5 - Ouvrages portuaires, côtiers et offshore

Tableau 2. Synthèse des essais mécaniques en vraie grandeur.

\begin{tabular}{|c|c|c|c|c|c|}
\hline $\begin{array}{l}\text { Nom } \\
\text { commercial }\end{array}$ & $\begin{array}{l}\text { Nom } \\
\text { botanique }\end{array}$ & $f_{m, k}$ & $E_{m, m}$ & $\rho_{m, 12 \%}$ & $\begin{array}{l}\text { Classe } \\
\text { EN338* }\end{array}$ \\
\hline Angelim & Dinizia excelsa & 60.4 & 22084 & 1082 & D60 \\
\hline Cupiuba & Goupia glabra & 53.1 & 21414 & 822 & D50 \\
\hline Eveuss & Klainedoxa gabonensis & 51.0 & 20998 & 1019 & D50 \\
\hline Okan & Cylicodiscus gabunensis & 47.3 & 19318 & 998 & $D 40$ \\
\hline Tali & Erythrophleum ivorense & 40.5 & 17200 & 815 & D35 \\
\hline Azobé* & Lophira alata & 70 & 20000 & 1080 & $D 70$ \\
\hline Greenheart* & Chlorocardium rodiei & 70 & 20000 & 1080 & $D 70$ \\
\hline Balau* & Shorea spp. & 70 & 20000 & 1080 & $D 70$ \\
\hline Opepe* & Nauclea diderrichii & 50 & 14000 & 780 & D50 \\
\hline Pin Douglas* & Pseudotsuga menziesii & 24 & 11000 & 420 & D24 \\
\hline Karri* & Murraya koenigii & 50 & 14000 & 780 & D50 \\
\hline \multicolumn{6}{|c|}{$\begin{array}{l}\text { Légende }: f_{m, k}=\text { résistance à la flexion dépassée par 95\% des prises d'essai en }\left(\mathrm{N} / \mathrm{mm}^{2}\right), E_{m, m}=\text { Valeur moyenne } \\
\text { du module élastique en }\left(\mathrm{N} / \mathrm{mm}^{2}\right) \text { et } \rho_{m, 12 \%}=\text { masse volumique moyenne corrigée à } 12 \% \text { d'humidité }\left(\mathrm{kg} / \mathrm{m}^{3}\right) \\
\text { Note } * * \text { à titre d'information, il faut noter EN } 1912 \text { donne des exemple d'essences pour ces classes comme le } \\
\text { chêne }=D 30 \text {, le teak }=D 40 .\end{array}$} \\
\hline
\end{tabular}

Tableau 3. Synthèse des appréciations qualitatives réalisées lors des essais de mise en ouvre.

\begin{tabular}{|c|c|c|c|c|c|c|}
\hline $\begin{array}{l}\text { Nom } \\
\text { commercial }\end{array}$ & $\begin{array}{l}\text { Nom } \\
\text { botanique }\end{array}$ & Référence & Eclats & $\begin{array}{l}\text { Facilité } \\
\text { de sciage }\end{array}$ & $\begin{array}{l}\text { Facilité } \\
\text { de } \\
\text { mortaisage }\end{array}$ & Commentaires \\
\hline \multirow{3}{*}{ Angelim } & \multirow{3}{*}{$\begin{array}{l}\text { Dinizia } \\
\text { excelsa }\end{array}$} & Greenheart & $\approx$ & - & + & \multirow{3}{*}{$\begin{array}{l}\text { Malodorant } ; \\
\text { globalement facile à } \\
\text { travailler }\end{array}$} \\
\hline & & Azobé & $\approx$ & - & + & \\
\hline & & Chêne & + & - & - & \\
\hline \multirow{3}{*}{ Cupiuba } & \multirow{3}{*}{$\begin{array}{l}\text { Goupia } \\
\text { glabra }\end{array}$} & Greenheart & + & $\approx$ & + & \multirow{3}{*}{$\begin{array}{l}\text { Peu odorant ; peu } \\
\text { contenir des zones } \\
\text { plus tendre }\end{array}$} \\
\hline & & Azobé & - & + & + & \\
\hline & & Chêne & - & - & - & \\
\hline \multirow{3}{*}{ Eveuss } & \multirow{3}{*}{$\begin{array}{l}\text { Klainedoxa } \\
\text { gabonensis }\end{array}$} & Greenheart & $\approx$ & + & + & \multirow[t]{3}{*}{ Grain irrégulier } \\
\hline & & Azobé & $\approx$ & $\approx$ & + & \\
\hline & & Chêne & + & - & - & \\
\hline \multirow{3}{*}{ Okan } & \multirow{3}{*}{$\begin{array}{l}\text { Cylicodiscus } \\
\text { gabunensis }\end{array}$} & Greenheart & - & + & + & \multirow{3}{*}{$\begin{array}{l}\text { Grain très } \\
\text { irrégulier mais facile } \\
\text { à travailler }\end{array}$} \\
\hline & & Azobé & $\approx$ & + & + & \\
\hline & & Chêne & + & $\approx$ & - & \\
\hline \multirow{3}{*}{ Tali } & \multirow{3}{*}{$\begin{array}{l}\text { Erythrophleu } \\
\text { m ivorense }\end{array}$} & Greenheart & - & + & + & \multirow{3}{*}{$\begin{array}{l}\text { Grain grossier : bon } \\
\text { état de surface } \\
\text { difficile à obtenir }\end{array}$} \\
\hline & & Azobé & - & + & + & \\
\hline & & Chêne & $\approx$ & - & + & \\
\hline
\end{tabular}

\subsection{Réalisation d'un pré-pilote}

Pour confirmer les tendances observées à partir des essais présentés plus haut, il sera nécessaire de mettre ces essences en situation. Avant que l'ensemble des essais ne soient terminés, un chantier de réfection d'un épi à Pevensey (cote sud de l'Angleterre) a servi de pilote préliminaire afin de mettre au point une méthode d'implantation et de suivi des essences. Deux grandes volets seraient à étudier: l'aptitude au battage des essences en particulier pour l'utilisation en pieu; la résistance à l'usure et aux 
xylophages. Le site de Pevensey a permis de tester la méthodologie pour le $2^{\text {ème }}$ aspect sachant que la structure de l'épi était encore intègre. Ainsi, la plaquage de l'épi a été remplacé par une combinaison d'essences alternatives. En l'occurrence, il s'agit de tali, niove, souge et balau en plus de greenheart car l'ensemble des essais préliminaires ( $\mathrm{cf}$ 2) n'était pas terminé. Il a été jugé que le risque de mettre en place des essences qui pourraient mal résister était acceptable sachant que l'ensemble des épis de plage feront ultérieurement l'objet d'une réfection complète qui permettrait de revenir réparer.

Ainsi, un plan d'implantation a été développé (voir figure 5) qui permet d'exposer les espèces à des niveaux différents de sollicitation par la plage (pour l'abrasion), à des conditions différentes d'eau et d'humidité (pour les xylophages) tout en ayant une essence de référence (ici le greenheart) à proximité pour juger de manière relative de la performance. Il a aussi été jugé que la mise en œuvre de "blocs témoins" n'était pas une bonne solution car elle conduirait à la création de "niches" trop favorables aux xylophages, ainsi que des "saillants" qui pourraient conduire à une usure locale exacerbée.

Enfin, il a été décidé que le meilleur moyen de suivre l'évolution était :

a) de faire des estimations visuelles par des spécialistes de l'invasion et par l'attaque par les xylophages ;

b) de mesurer régulièrement ( 2 fois dans l'année) l'épaisseur des planches de plaquage à l'aide d'un pied à coulisse.

Ces travaux préliminaires ont été réalisés cours de l'année 2008 et font actuellement l'objet d'un suivi qui permettra de valider le principe de suivi sur des ouvrages pilotes à construire en 2010 et dont le suivi devrait être de 5 à 7 ans.

\section{Conclusion}

L'utilisation de ressources renouvelables et durables pour la construction des ouvrages maritimes et côtiers est un objectif accessible notamment grâce au bois. Cependant cela n'est possible qu'en s'assurant de l'origine légale et diversifiée du bois ainsi que d'une exploitation durable des forêts d'origine.

Jusqu'à présent, les travaux maritimes et côtiers ont majoritairement utilisés et par défaut l'azobé et le greenheart parce qu'elles présentent de bonnes caractéristiques géométriques, mécaniques et de durabilité, en particulier face au risque d'attaque par les xylophages et au risque d'usure. Cela accroît la pression environnementale et commerciale sur ces seules espèces.

Le travail présenté ici a permis d'identifier une trentaine d'essences pouvant être des alternatives à l'azobé et au greenheart. Une vingtaine a été soumise à des essais accélérés de durabilité. Ces résultats permettent notamment de choisir des essences par rapport au risque prévalent sur le site.

Une analyse multicritère a permis d'isoler cinq essences alternatives que sont angelim vermelho (dinizia excelsa), cupiuba (goupia glabra), evuess (klainedoxa gabonensis), 
okan (Cylicodiscus gabunensis), tali ou missanda (erythrophleum ivorense). Pour ces essences, l'essentiel des essais normés requis pour l'utilisation dans les ouvrages ont été conduits en vraie grandeur. Ceci fournit une base essentielle permettant maintenant l'utilisation de ces essences alternatives pour lesquelles aucune référence n'était disponible, permettant ainsi de diminuer la pression sur l'azobé et le greenheart.

La réalisation de pilotes pour confirmer la performance réelle de ces essences en service est en cours et s'appuie sur une pré-pilote réalisé lors de la reconstruction d'un épi sur la côte sud de l'Angleterre.

\section{Remerciements}

Le comité de pilotage de ce travail a été animé par M Meadon de Environment Agency et le travail a été piloté par S Dupray du CETMEF (à HRWallingford au moment du projet). Les travaux ont été menés par HRWallingford et TRADA. Le projet a été financé par the Environment Agency, TRADA, British Waterways, Crown Estate, Volker Stevin et CETMEF. Les échantillons ont été gracieusement fournis par EcoChoice et Aitken \& Howard. Les essais in situ et en atelier ont été gracieusement effectués par Mackley Construction et British Waterways. Le site pilote préliminaire a été accueilli par Bournemouth City Council qui a pris en charge l'achat des matériaux pour l'épi pilote préliminaire.

\section{Références}

AITKEN \& HOWARD (2007). List of commercially available hardwood timber that could form suitable alternative to greenheart and ekki, $A \& H$. Personal communication. BIODIVERSITY INTERNATIONAL (2007). Study into alternatives to greenheart and ekki tropical hardwood timber. Environment Agency, Unpublished report, pp 1-40.

BOLZA E., KEATING W.G. (1972). African timbers - the properties, uses and characteristics of 700 species. Division of Building Research, Commonwealth Scientific and Industrial Research Organisation (Australia).

BORGES L.M.S., CRAGG S.M., WILLIAMS J.R. (2003). Comparing the resistance of a number of lesser known species of tropical hardwoods to the marine borer Limnoria using a short term laboratory assay. International Research Group on Wood Preservation, Report IRG/WP 03.

BRAZIER J. (1995). Greenheart and the choice of timbers for marine work. Notes from the Half day meeting at the maritime board of the Institution of Civil Engineers on the $3^{\text {rd }}$ October, pp 1-12.

CHUDNOFF M. (1979). Individual data sheets for species by region of origin: Africa: Tropical timbers of the world. Forest Products Laboratory, Forest Services, United States Department of Agriculture.

CROSSMAN M., SIMM J.D. (2004). Manual on the use of timber in coastal and river engineering. Thomas Telford, London. 
DUPRAY S.W., SIMM J.D. (2007). Climate change : materials and material uses. The edge - The magazine of coastnet, winter edition, pp 14-15.

ECOCHOICE (2007). List of commercially available hardwood timber that could form suitable alternative to greenheart and ekki, ECOCHOICE, 2007. Personal communication.

GOLLASH S. (2006). Toredo navalis - data sheet. EU project DAISIE.

GREEN M., PITMAN A.J. (2001). A preliminary study of timber susceptibility to attack by the weevil Euophryum confine (Broun). Proceeding of the 5th International Conference on the Development of Wood Science, Wood Technology and Forestry, Ljubljana, Slovenia.

HALL G.S., SAUNDERS R.G. (1967). Incidence of marine borers around Britain's Coast. Timber Research and Development Association, High Wycombe, Report $\mathrm{B} / \mathrm{RR} / 4$.

MAINIERI C., CHIMELO J.P. (1989). Fichas de caracteristicas das Madeiras Brasileiras. Instituto de Pesquisas Tecnologicas Divisăo de Madieras, Săo Paulo.

MAUN K. (2000). Handbook of Hardwoods. Building Research Establishment Press.

METTEM C.J., RICHENS A.D. (1991). Hardwoods in construction. Timber Research and Development Association, High Wycombe.

NEWTON A., MUDGE S.M. (2003). Temperature and salinity regimes in a shallow, mesotidal lagoon, the Ria Formosa, Portugal. Estuarine, Coastal and Shelf Science, Vol. 57, pp 73-85. doi:10.1016/S0272-7714(02)00332-3

NGUYEN M.N., LEICESTER R.H., WANG C. (2008a). Marine Borer Attack on Timber Structures - MANUAL NO. 7. Commonwealth Scientific and Industrial Research Organisation, Vistoria (Australia).

NGUYEN M.N., LEICESTER R.H., WANGA C.H., COOKSONB L.J. (2008b). Probabilistic procedure for design of untreated timber piles under marine borer attack. Reliability Engineering \& System Safety, 93, No. 3, pp 482-488. doi:10.1016/j.ress.2006.12.012

NICHOLLS R.J., DE LA VEGA-LEINERT A.C. (2008). Implications of sea-level rise for Europe's coasts: an introduction. Journal of Coastal Research, 24, No 2, pp 285-287. doi:10.2112/07A-0002.1

OLIVER A.C. (1974). Timber for marine and fresh water construction. Timber Research and Development Association, High Wycombe.

RAPP A., BRISCHKE C., WELZBACHER C.R. (2007). The influence of different soil substrates on the service life of Scots pine sapwood and oak heartwood in ground contact. Wood Material Science and Engineering, 2, No 1, pp 15-21. doi:10.1080/17480270701273015 
Thème 5 -Ouvrages portuaires, côtiers et offshore

SAWYER G.S., WILLIAMS J.R. (2005). An investigation to assess the feasibility of developing an accelerated laboratory test to determine the abrasion resistance of lesser-used timber species for use in marine construction. International Research Group on Wood Preservation, Report IRG/WP 05.

SIMM J.D., WALLIS M.J., COLLINS K.J., ATKINS R. (2004). Re-use of materials in coastal and river engineering. Proceedings of the Institution of Civil Engineers, Engineering sustainability, 157, No 3, pp 113-121. doi:10.1680/ensu.2004.157.3.113

WILLIAMS J.R. (1999). Factors affecting the performance of antisapstain preservatives in the field. $\mathrm{PhD}$ thesis, Department of Biology, Imperial College, London.

WILLIAMS J.R., SAWYER G.S., CRAGG S.M., SIMM J.D. (2004a). A questionnaire survey to establish the perceptions of UK specifiers concerning the key material attributes of timber for use in marine and fresh water construction. International Research Group on Wood Preservation, IRG/WP 04.

WILLIAMS J.R., CRAGG S.M., BORGES L.M.S., ICELY J.D. (2004b). Marine exposure assessment of the natural resistance of a number of lesser known species of tropical hardwoods to teredinid and limnoriid borers. International Research Group on Wood Preservation. 